\title{
The scaling of syntactic relatedness: A new method in psycholinguistic research
}

\author{
W. J. M. LEVELT, University \\ Groningen, The Netherlands
}

Subjects were requested to judge the degrees of syntactic relation for all pairs of words from a sentence. If it is hypothesized that the judgments are determined mainly by the deep phrase structure of the sentence, it is possible to map the relatedness data on comprehensive tree graphs. Various examples are given.

The purpose of this paper is to present a scaling method that may be of some use for the psychology of grammar. The method is a judgment procedure, called judgment of syntactic relatedness. It can be supplemented by several types of data analysis. In this paper, we will limit ourselves to one: hierarchical clustering analysis.

The type of judgment under concern can be illustrated by the following example. Given the sentence The too expensive food was tasteless, one can ask Ss which pair of words is more related, the and too or the and food. Nobody hesitates to choose the latter pair. People have rather explicit and consistent opinions on such matters. The introspective evidence involved is not essentially different from "grammaticality," "paraphrase," and other intuitive notions on which modern grammars are based. It is also apparent from the example that syntactic structure is a determinant of such judgments. The present paper describes some exploratory experiments leading to the formulation of two hypotheses with respect to the relation between syntactic structure and relatedness judgments (for further details, see Levelt, 1967. 1969a. b).

The main purpose of hierarchical clustering analyses of word relatedness data is to obtain faithful geometric representations that allow a direct assessment of grammatical factors underlying the judgments. It should be kept in mind that such representations are not hypothesis-testing devices. They are merely given to visualize certain aspects of the data that are of grammatical interest.

\section{EXPERIMENT 1 Method}

The Ss were 24 Harvard and Radcliffe students (no language students). They were presented with the typewritten sentence, the boy has lost a dollar. They were instructed to judge the degrees of reports. Here we want to proceed a little further by considering a case of coordination.

\section{EXPERIMENT 2}

Method

Eight Groningen University students (no language students) were instructed to make relatedness judgments on the sentence, Jan eet $_{1}$ appels en Piet eet ${ }_{2}$ peren. (English: John eats apples and Peter eats pears.) Ss were presented with the typewritten sentence as well as with a list of all pairs of words from the sentence. Each pair was accompanied by a 7-point relatedness scale. The word order in the pairs and the order of pairs were randomized for each $S$. Ss judged by encircling 1 of the 7 points of each scale.

\section{Results}

The average scale value ( 7 for "strongly related") for each word pair is given in the right upper half of Table 2 .

Analysis and Discussion

The result of a clustering analysis is given in Fig. 2. Again, a satisfactory structure is obtained. The two sentences that are coordinated turn up as independent structures with identical topologies. The coordinator (and) is least related to any other word. Subject-verb clusterings often occur for so-called middle verbs (verbs with an optional object). This is not surprising from the standpoint of a case grammar.

The sentence is also interesting because the second eats is deletable: John eats apples and Peter pears. How will this sentence be judged, by surface relations or by underlying grammatical relations? In the latter case, we should find that the relation eats, apples will be about equal to the relation eats, pears. If this happens, a tree representation will break down, because the hierarchy can only represent one of these relations at a time.

\section{EXPERIMENT 3}

Method

A new group of eight Ss judged the sentence Jan eet appels en Piet peren (John eats apples and Peter pears), as in Experiment 2.

Results and Discussion

Average scale values are given in Table 2, lower half. A clustering analysis resulted in a confused structure. It can be summarized

Table 1

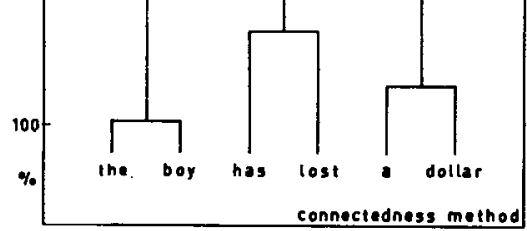

Relatedness Values for the boy has lost a dollar

\begin{tabular}{lcccccc}
\hline Words & 1 & 2 & 3 & 4 & 5 & 6 \\
\hline 1 the & -- & .99 & .43 & .29 & .19 & .16 \\
2 boy & & -- & .63 & .65 & .16 & .31 \\
3 has & & & -- & .86 & .31 & .40 \\
4 lost & & & & -- & .42 & .70 \\
5 a & & & & -- & .94 \\
6 dollar & & & & & -- \\
\hline
\end{tabular}


by the following bracketing: (/(/John eats) apples) pears) Peter) and). This is indeed the result of the fact that eats and apples and eats and pears are about equally related (6.1 and 5.9, respectively). This brings us to the formulation of a second hypothesis: Relatedness judgments are mainly determined by the underlying structure of the sentence. The following experiment is designed to give further confirmation of this hypothesis. Moreover, an analysis procedure is proposed that, assuming that the second hypothesis is correct, can yield adequate tree representations of the data, even in cases of deletion.

\section{EXPERIMENT 4 Method}

All 16 Ss of the previous two experiments judged the sentence Carla pakt het boek en gaat naar school (Carla takes the book and goes to school) by the same 7 -point rating procedure. This is a coordinated sentence with the second Carla deleted.

\section{Results and Analysis}

In accordance with the second hypothesis, the pairs Carla, takes and Carla, goes are not significantly different in relatedness values $(6.3$ and 6.1 , respectively). Predictably, this fact is not apparent from the obtained clustering, in bracketed form: (//Carla takes) ((goes to) school) the book) and). In such cases, a faithful representation of the data can be obtained by expanding the data matrix by a dummy row and column for the deleted word. For the present case, the expanded matrix is given in Table 3. The Carla values are the original Carla values, except for the relations to goes, to, and school, which were put to the minimum value (1). Symmetrically, the $\mathrm{Carla}_{2}$ values are the original values, except for the relations to takes, the, and book. Together with the $\mathrm{Carla}_{1}, \mathrm{Carla}_{2}$ relation, they were given the

Table 2

Relatedness Values for John eats apples and Peter (eats) pears. Uppe Half: Experiment 2, Lower Half: Experiment 3 .

\begin{tabular}{lccccccc}
\hline Words & 1 & 2 & 3 & 4 & 5 & 6 & 7 \\
\hline 1 John & -- & 6.9 & 6.0 & 3.0 & 4.5 & 1.1 & 1.3 \\
2 eats & 6.5 & -- & 6.3 & 2.0 & 1.3 & 4.0 & 1.3 \\
3 apples & 5.3 & 6.1 & -- & 2.1 & 1.3 & 1.3 & 4.1 \\
4 and & 1.4 & 1.9 & 1.4 & -- & 2.4 & 1.9 & 2.0 \\
5 Peter & 4.4 & 5.6 & 1.3 & 4.3 & -- & 7.0 & 5.8 \\
6 (eats) & -- & -- & --1 & -- & -- & -- & 5.6 \\
7 pears & 1.9 & 5.9 & 4.1 & 3.0 & 5.5 & -- & -- \\
\hline
\end{tabular}

Table 3

Expanded Relatedness Matrix for Carla takes the book and goes to school Added Values are Marked by *.

\begin{tabular}{llllllllll}
\hline Words & 1 & 2 & 3 & 4 & 5 & 6 & 7 & 8 & 9 \\
\hline 1 Carla 1 & -- & $1.0^{*}$ & 6.3 & 1.4 & 4.4 & 2.0 & $1.0^{*}$ & $1.0^{*}$ & $1.0^{*}$ \\
2 Carla2 & & -- & $1.0^{*}$ & $1.0^{*}$ & $1.0^{*}$ & 2.0 & 6.1 & 2.9 & 4.5 \\
3 takes & & & -- & 3.4 & 5.6 & 2.5 & 3.4 & 1.2 & 1.2 \\
4 the & & & & -- & 6.9 & 1.3 & 1.1 & 1.1 & 1.1 \\
5 book & & & & & -- & 1.3 & 1.1 & 1.1 & 2.9 \\
6 and & & & & & & -- & 3.3 & 1.5 & 2.1 \\
7 goes & & & & & & & -- & 6.6 & 5.0 \\
8 to & & & & & & & -- & 6.4 \\
9 school & & & & & & & & & --
\end{tabular}

value 1 . Though the double role of Carla in the underlying structure is in this way imposed on the expanded data matrix, this is in itself not sufficient for obtaining a satisfactory clustering. The result in Fig. 3 is, however, not only grammatically meaningful, but is also a faithful representation of the original relatedness data.

\section{DISCUSSION}

It should be noted that underlying phrase structure is not the sole determinant of relatedness judgments. Words similar in meaning and adjacent words tend to have slightly inflated relatedness values. Also, other variables like order effects need further study.

Relatedness judgments can be another source of empirical grammatical evidence. The two hypotheses proposed in this paper are a first step in establishing the relation between such judgments and linguistic structure. As in the case of acceptability judgments and their relation to linguistic grammaticality, a performance theory is required if relatedness judgments are to be used in the construction of grammars. REFERENCES

JOHNSON, S. C. Hierarchical clustering schemes. Psychometrika, 1967, 32, 241-254.

LEVELT, W. J. M. Psychological representations of syntactic structure. (Report HB 69-37 EX 1967, Department of Psychology, Groningen University.) In T. G. Bever and W. Weksel (Eds.), The structure and psychology of language. New York: Holt, Rinehart \& Winston, in press.

LEVELT, W. J. M. The perception of syntactic structure. (Report HB 69-30 EX, Department of Psychology, Groningen University.) Invited paper presented at the XIX International Congress of Psychology, London, 1969a.

LEVELT, W. J. M. The scaling of syntactic relationships. (Report HB 69.37 EX, Department of Psychology, Groningen University.) Paper read at The Psycholinguistics Conference, Bressanone, $1969 \mathrm{~b}$.

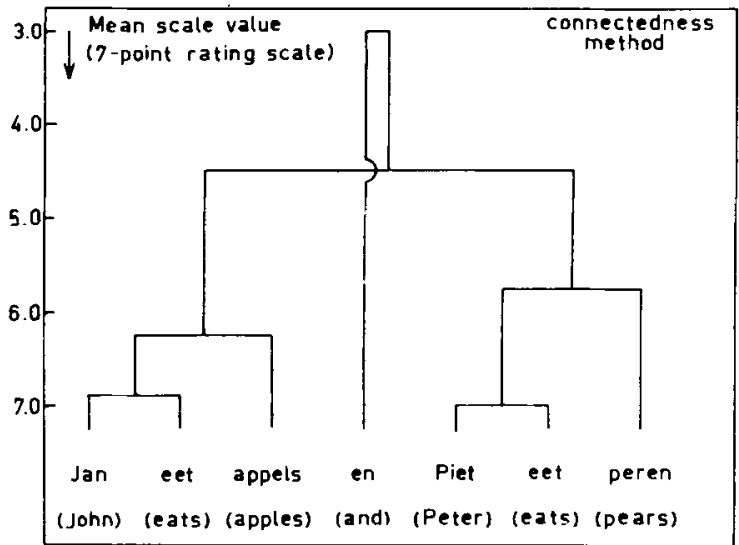

Fig. 2. Tree graph for John eats apples and Peter eats pears.

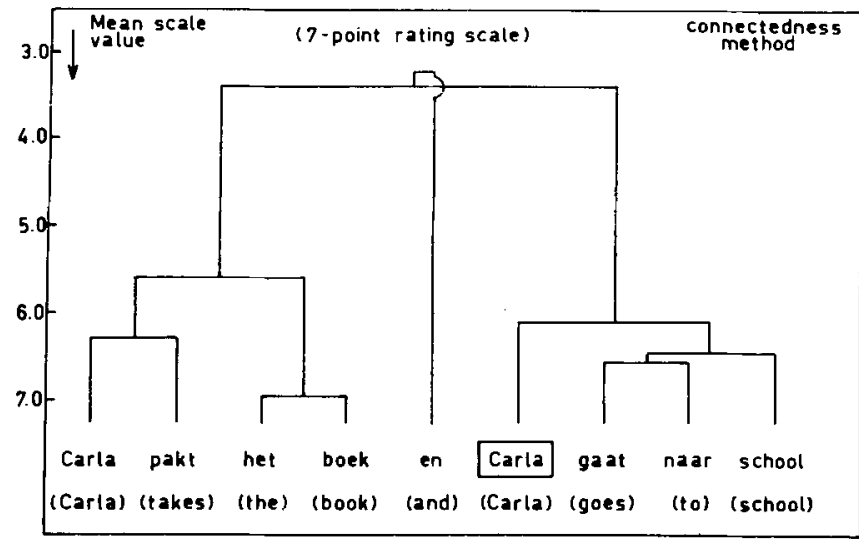

Fig. 3. Tree graph for Carla takes the book and goes to school, with addition of deleted element Carla. 